\title{
Powder-Metallurgical Process for Producing Metallic Microchannel Devices
}

\author{
Tatsuya Ohmi, Masashi Takatoo*, Manabu Iguchi, Kiyotaka Matsuura and Masayuki Kudoh \\ Division of Materials Science and Engineering, Graduate School of Engineering, \\ Hokkaido University, Sapporo 060-8628, Japan
}

We investigated a simple and economical method for producing free-form microchannels in metal bodies. The concept for our process is based on a microscopic infiltration phenomenon that often occurs during liquid phase sintering of a mixture of metal powders with different melting points. A shaped compound of the metal powder with lower melting point and an organic binder are used as the sacrificial core that gives the shape of the microchannel. A body-metal powder compact that includes the sacrificial core is sintered at a temperature between the melting points of the sacrificial-core metal and body metal. The organic binder is removed during heating of the powder compact, and infiltration of molten sacrificial-core metal into the body-metal powder produces a microchannel and a lining layer. We examined following combinations of metal powders: titanium-aluminum, nickel-aluminum, copper-tin, and iron-copper. Metallographic observations confirmed that microchannels were produced in the metallic bodies in all these systems. Furthermore, in the case of the titanium body metal with an Al-Cu alloy sacrificial-core metal, the inner wall of the microchannel was smoother than the case of titanium with aluminum. The copper content of the sacrificial-core metal influenced the composition and structure of the microchannel lining. [doi:10.2320/matertrans.47.2137]

(Received February 28, 2006; Accepted May 8, 2006; Published September 15, 2006)

Keywords: microchannel, powder metallurgy, infiltration, intermetallic compound, functional-material lining

\section{Introduction}

A microchannel is a thin channel with a characteristic dimension of several tens or hundreds of micrometers. Remarkable progress has been made in recent years in microchannel device technology. ${ }^{1,2)}$ Metallic microchannel devices have attracted interest for small-scale heat exchangers or microreactors for highly exothermic reactions. A detailed review of microchannel heat sinks was presented by Phillips. ${ }^{2)}$ Papautsky et al. described a low-temperature process for surface fabrication of metallic microchannels on a silicon or glass substrate using electroplating and photolithography. ${ }^{3)}$ Rebrov et al. reported their study on the activity and heat transfer characteristics of several microreactors for the ammonia oxidation reaction, which is a strongly exothermic reaction. ${ }^{4)}$

One of the most important topics in the study of metallic microchannel devices is configuration of a method to fabricate microchannels in metal bodies. Some microchanneling processes have been proposed, including precision mechanical machining, such as micro-end milling or microturning, photoetching, micro-electro discharge machining, laser or electron beam machining, and galvanization., ${ }^{5,6)}$ All these processes are methods of manufacturing structured surfaces. However, methods to fabricate microchannels directly in bulk metals would be more beneficial from a practical standpoint.

We recently investigated a powder-metallurgical microchanneling process using a metal powder and a wire of another metal as raw materials.,8) The concept for the process is based on a microscopic infiltration phenomenon that often occurs during liquid-phase sintering of a powder mixture of different metals, e.g., a mixture of titanium and aluminum. A thin aluminum wire with a controlled shape is used in this microchanneling process instead of aluminum powder. Figure 1 illustrates the supposed microchanneling

*Graduate Student, Hokkaido University

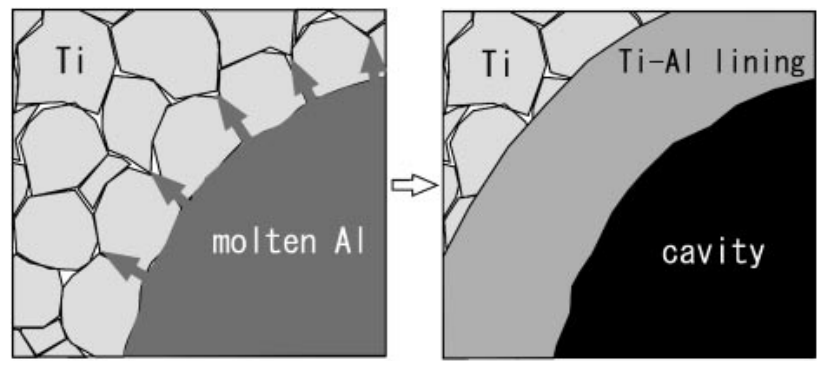

Fig. 1 Microchannel formation mechanism.

mechanism. A titanium-powder compact containing shaped aluminum wire is sintered at a temperature above the melting point of aluminum. Liquid aluminum that originates from the wire flows into narrow capillaries between the titanium powder particles during sintering of the powder compact and reacts with the titanium particles to produce $\mathrm{Ti}-\mathrm{Al}$ intermetallic layers that surround the cavity formed at the site initially occupied by the aluminum wire. Thus, the microscopic reactive infiltration generates the formation of a microchannel that is the same shape as the original wire. Alternatively, the aluminum wire acts as a sacrificial core that gives the shape of the microchannel and as an aluminum source for the resulting intermetallic lining.

Figure 2 shows the structure and aluminum-concentration profile in a lining layer produced in a Ti-Al specimen. The concentration ranges of the equilibrium phases at the sintering temperature, $1473 \mathrm{~K}$, are also shown in the figure. The lining layer is constructed in a concentric configuration and consists of three sub-layers. The aluminum concentration in the innermost sub-layer (from $x=0$ to $100 \mu \mathrm{m}$ ) is in the range from 35 to $63 \mathrm{~mol} \%$. In a Ti-Al binary alloy phase diagram, ${ }^{9)}$ the equilibrium phases in the concentration range are alpha-titanium solid solution and $\mathrm{TiAl}$ at the sintering temperature $1473 \mathrm{~K}$, and those at room temperature are $\mathrm{Ti}_{3} \mathrm{Al}, \mathrm{TiAl}$ and $\mathrm{TiAl}_{2}$. For the second sub-layer (from $x=$ 


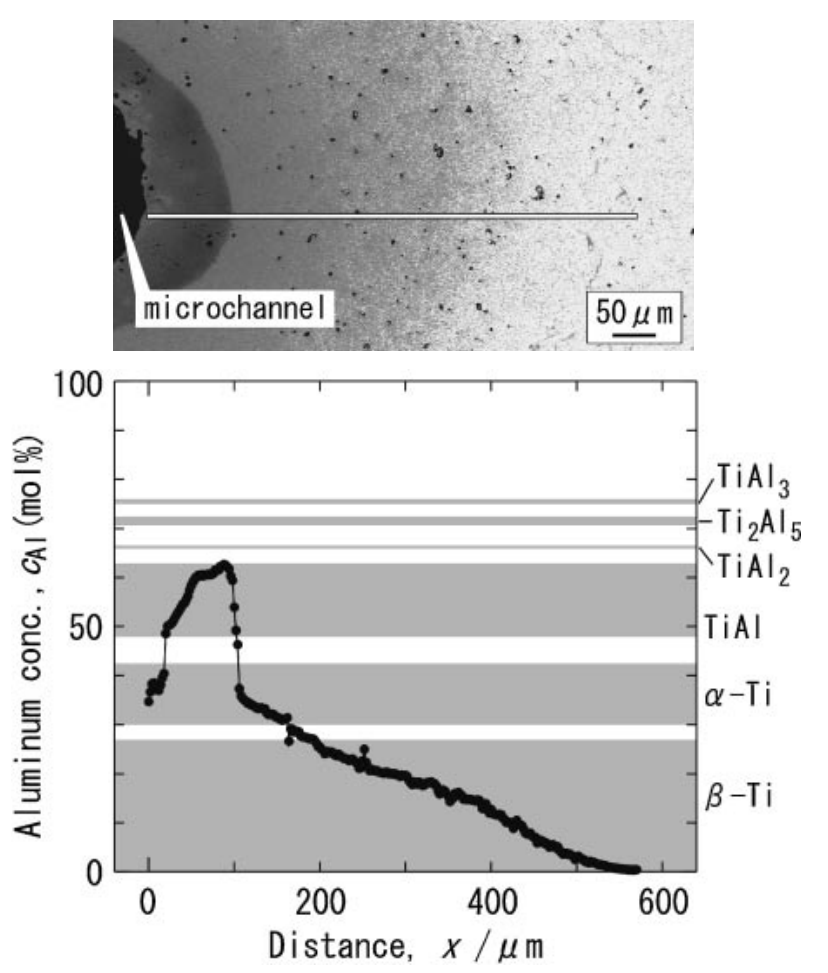

Fig. 2 Structure and aluminum-concentration profile in a lining layer formed in a Ti-Al specimen produced from titanium powder and aluminum wire. ${ }^{7)}$ The gray bands described in the concentration-profile graph show the concentration ranges of the equilibrium phases at the sintering temperature, $1473 \mathrm{~K}$.

100 to $200 \mu \mathrm{m}$ ), the aluminum concentration range is from 25 to $35 \mathrm{~mol} \%$ and the corresponding phases are alpha-titanium and beta-titanium at $1473 \mathrm{~K}$, and $\mathrm{Ti}_{3} \mathrm{Al}$ and $\mathrm{TiAl}$ at room temperature. For the third sub-layer (from $x=200$ to 400 $\mu \mathrm{m})$, the aluminum concentration range is from about 15 to $25 \mathrm{~mol} \%$. The equilibrium phase at $1473 \mathrm{~K}$ is beta-titanium. On the other hand, the equilibrium phases at room temperature are alpha-titanium and $\mathrm{Ti}_{3} \mathrm{Al}$. It is noteworthy that the first layer is in close contact to the second layer, and a graded concentration profile is observed in the area from the second layer to the titanium matrix.

Various alloys and intermetallic compounds have been considered as functional materials. Microchannel devices with a functional lining may have numerous new uses.

However, this process has the following crucial limitations.

(1) Precise bending and bonding of the wire are required to fabricate a microchannel network with a complicatedly diverged configuration.

(2) The sacrificial-core metal is limited to metal that can be formed into a wire.

(3) The cross-sectional configuration and size of the microchannel cannot be changed gradually or locally.

(4) It is difficult to control the distribution of alloying elements in the sacrificial core. Therefore, this process cannot control the composition or structure of the microchannel lining by addition of a third element.

We proposed a new microchanneling process to overcome above difficulties. ${ }^{10)}$ A shaped compound of a metal powder and an organic binder are used as the sacrificial core in our

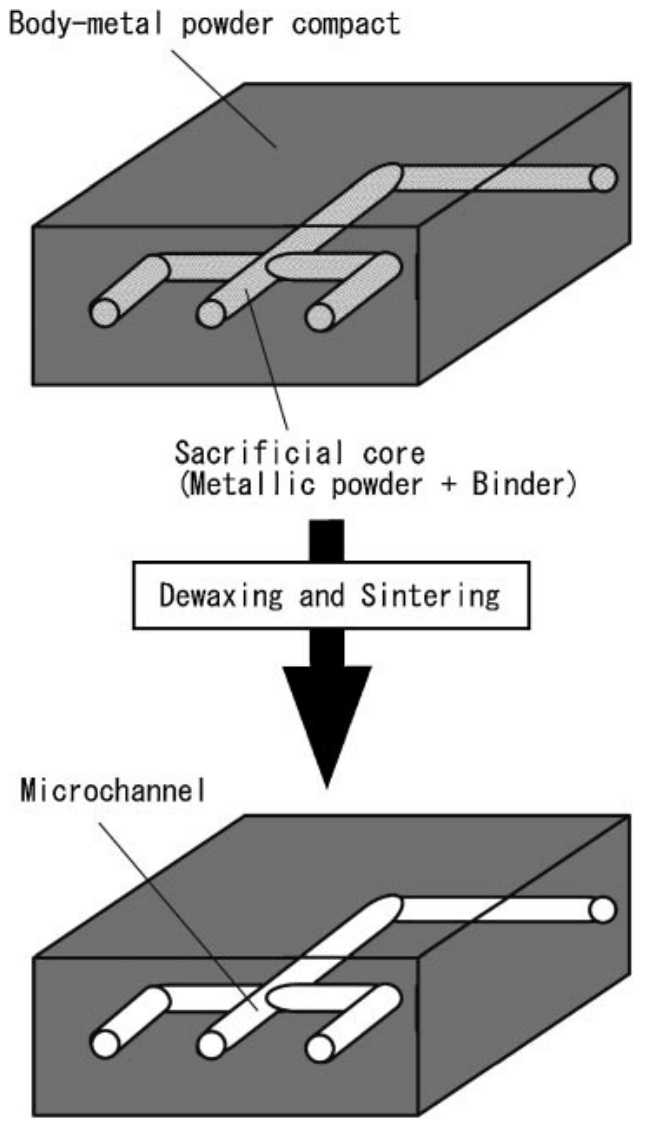

Fig. 3 Schematic illustration of the concept of the free-form microchanneling process.

process instead of metal wire. A powder-binder compound with plasticity or fluidity is formed into the intended shape by a micro-injection process. Configuration of the process is similar to inkjet printing or pen plotting.

An alloy powder or a mixed powder containing a third element can be used as the sacrificial-core metal in our freeform microchanneling process. Therefore, control of the composition or structure of the microchannel lining will be possible.

In the present study, we examined the feasibility of the free-form microchanneling process with more practical conditions than the previous report. ${ }^{10)} \mathrm{We}$ also investigated the potential to realize a functional microchannel lining by third-element addition to the sacrificial-core metal.

\section{Experimental Procedure}

\subsection{Free-form microchanneling process}

Figure 3 presents an outline of the free-form microchanneling process. ${ }^{10)}$ The structural metal for the device is designated as the body metal in the figure, and the material that gives the shape of the microchannel is specified as the sacrificial core. The sacrificial core is produced from a mixture of an organic binder and a powder metal (sacrificialcore metal) with a lower melting temperature than the body metal.

In our experiments, a body-metal powder compact containing a shaped sacrificial core was sintered at a 
Table 1 Purity and size of the metal powders used for free-form microchanneling experiments.

\begin{tabular}{ccc}
\hline Metal & Diameter, $d / \mu \mathrm{m}$ & Purity, $c_{\mathrm{p}}(\operatorname{mass} \%)$ \\
\hline $\mathrm{Ti}$ & under 45 & 99 \\
$\mathrm{Ni}$ & 5 & 99.8 \\
$\mathrm{Cu}$ & $45-75$ & 99.9 \\
$\mathrm{Fe}$ & 45 & 99 \\
$\mathrm{Al}$ & 100 & 99.7 \\
$\mathrm{Sn}$ & 38 & 99.99
\end{tabular}

temperature between the melting points of the body metal and the sacrificial-core metal. We examined four combinations of body metal and sacrificial-core metal: (1) Titanium (body metal) and aluminum (sacrificial-core metal), (2) Nickel and aluminum, (3) Copper and tin, and (4) Iron and copper. Table 1 lists the purity and average particle size of each metal powder. The corresponding phase diagram ${ }^{11)}$ for each of the combinations (1), (2) and (3) indicates the possibility of formation of intermetallic compounds by a reaction between the body metal and the sacrificial-core metal. In contrast, only body-metal-based solid solutions form for the combination (4).

The organic binder was prepared from yellow wax and pine resin blended in a mass ratio of $1: 1$, which was mixed with a sacrificial-core metal powder in a given volume ratio (1:1) to produce the sacrificial-core compound. The compound was extruded into a wire $0.5 \mathrm{~mm}$ in diameter. Figures 4(a) and (b) depicts the wire and our extrusion equipment. The wire was cut to a $15 \mathrm{~mm}$ length for the sacrificial core.

A given mass of the body-metal powder $(6.5 \mathrm{~g}$ for titanium, $12.9 \mathrm{~g}$ for nickel, $13.2 \mathrm{~g}$ for copper and $11.3 \mathrm{~g}$ for iron), including the sacrificial core, was cold-pressed into a cylindrical green compact in a metal mold using unidirectional pressure. The green compact had a diameter of $20 \mathrm{~mm}$. Figure 5(a) schematically illustrates the green compact. Although the initial cross section of the sacrificial core was round, it was distorted into an oblate figure by unidirectional pressing, as depicted in Fig. 4(d). Table 2 provides the pressing pressure for each body metal and the height and relative density of the compact.

Figure 5(b) presents the heating patterns for the microchanneling heat treatment, including dewaxing and sintering. The organic binder was completely removed by the dewaxing heat treatment at $573 \mathrm{~K}$ for $3.6 \mathrm{ks}$. The sintering temperatures were $1473 \mathrm{~K}$ for the green compacts of $\mathrm{Ti}-\mathrm{Al}, \mathrm{Ni}-\mathrm{Al}$ and $\mathrm{Fe}-\mathrm{Cu}$ systems, and $1123 \mathrm{~K}$ for that of $\mathrm{Cu}-\mathrm{Sn}$ system. All the heat treatments were carried out in an argon gas atmosphere.

\subsection{Experiments on third-element effects}

We conducted several microchanneling experiments to investigate the effects of adding a third element to the sacrificial-core metal on the microchanneling behavior and the composition of the lining layer. Titanium was used for the body metal in these experiments, and the composition of the sacrificial-core metal was Al-17 mol\%Cu or Al-34 mol\%Cu. The $\mathrm{Al}-34 \mathrm{~mol} \% \mathrm{Cu}$ powder with an average diameter of

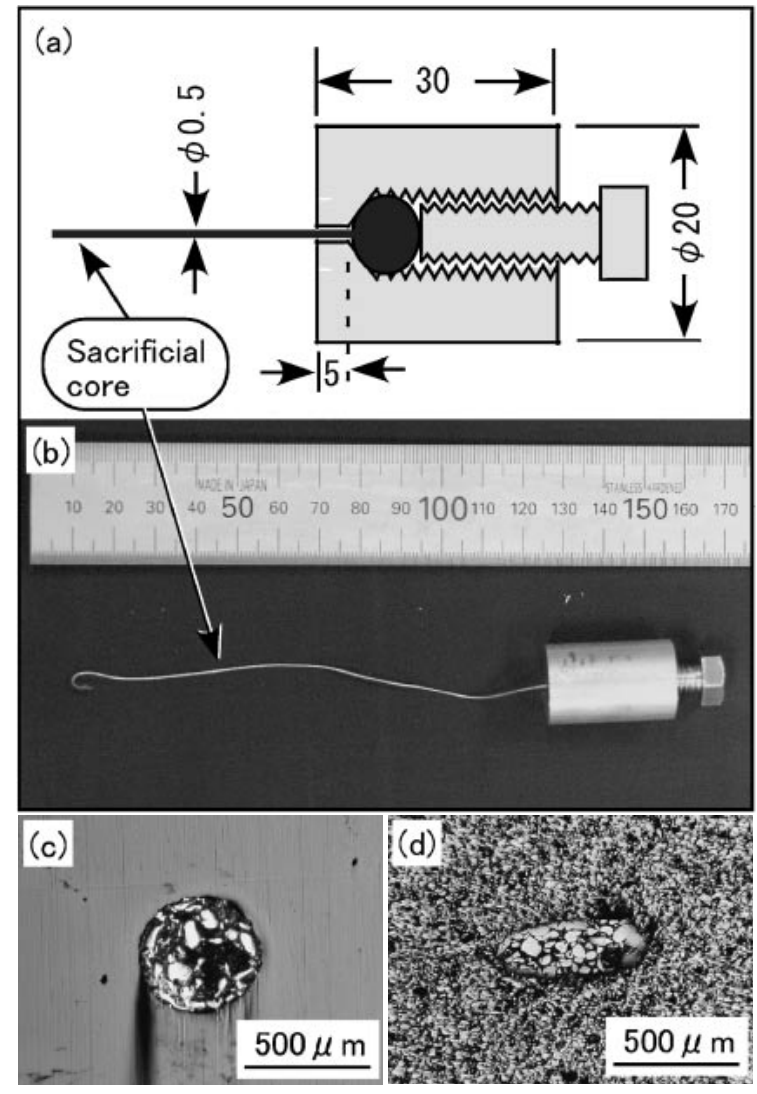

Fig. 4 Fabrication of the sacrificial core. (a) Schematic of the compoundextrusion equipment. (b) External appearance of the extrusion equipment with a filamentous sacrificial-core compound. (c) Optical micrograph of a cross section of the sacrificial core. (d) Optical micrograph of a cross section of the sacrificial core after compaction.

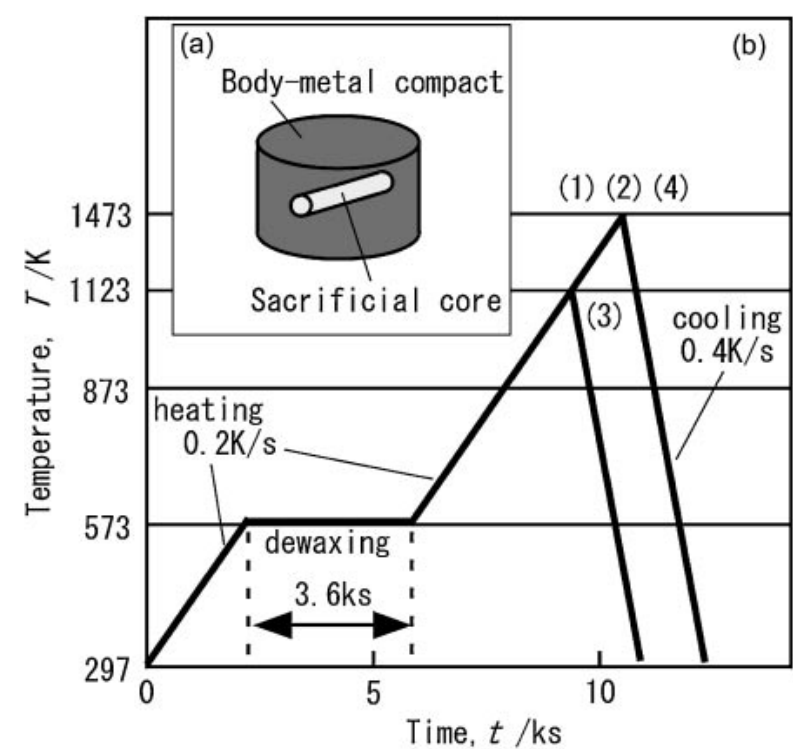

Fig. 5 Schematic of the body-metal powder compact with a sacrificial core (a) and time-temperature patterns for heat treatments (b). Combination of body metal and sacrificial-core metal: (1) Ti and Al, (2) Ni and Al, (3) $\mathrm{Cu}$ and $\mathrm{Sn}$, and (4) $\mathrm{Fe}$ and $\mathrm{Cu}$.

$35 \mu \mathrm{m}$ was produced by ingot casting and grinding. The Al$17 \mathrm{~mol} \% \mathrm{Cu}$ powder was prepared by mixing of the Al$34 \mathrm{~mol} \% \mathrm{Cu}$ powder and aluminum powder. 
Table 2 Pressing pressure, $P_{\mathrm{c}}$, resultant size (height, $H$ ) and relative density, $D_{\mathrm{r}}$, of powder compacts.

\begin{tabular}{cccc}
\hline Body metal & $P_{\mathrm{c}} / \mathrm{MPa}$ & $H / \mathrm{mm}$ & $D_{\mathrm{r}}(\%)$ \\
\hline $\mathrm{Ti}$ & 624 & 5.4 & 85 \\
$\mathrm{Ni}$ & 312 & 6.7 & 68 \\
$\mathrm{Cu}$ & 312 & 5.9 & 81 \\
$\mathrm{Fe}$ & 624 & 5.9 & 75 \\
\hline
\end{tabular}
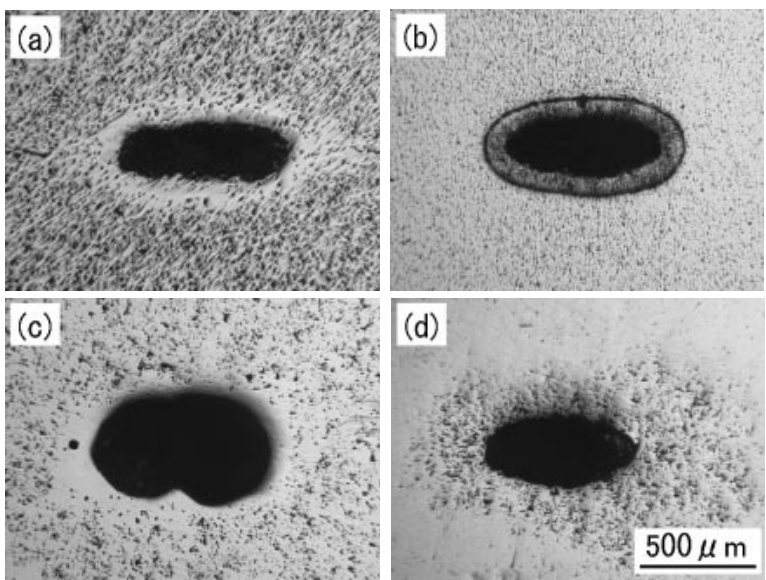

Fig. 6 Optical micrographs showing the formation of microchannels. Combination of body metal and sacrificial-core metal: (a) Ti and Al, (b) Ni and $\mathrm{Al},(\mathrm{c}) \mathrm{Cu}$ and $\mathrm{Sn}$, and (d) $\mathrm{Fe}$ and $\mathrm{Cu}$.

\section{Results and Discussion}

\subsection{Formation of microchannels}

Figure 6 presents examples of optical micrographs of cross sections of the microchannels fabricated by the free-form microchanneling process. Each microchannel was formed at the site initially occupied by the sacrificial core and was elliptical in shape in the cross section. The oblate shape of the contour resulted from deformation of the sacrificial core during press-forming of the green compact. A similar crosssectional shape was observed in the microchanneling experiments using titanium powder as the body-metal powder and aluminum wire as the sacrificial core. ${ }^{7,8}$ However, it is possible to fabricate a corrected initial shape of the sacrificial core in the free-form microchanneling process since the sacrificial-core compound can be formed easily.

\subsection{Constitution of Ti-Al lining layers}

Figure 7(a) contains a back-scattered electron image of the lining layer around the microchannel formed in a Ti-Al specimen. An EPMA line analysis result is provided in Fig. 7(b). The aluminum-concentration profile in Fig. 7(b) indicates that the maximum (about $45 \mathrm{~mol} \%$ or 30 mass \%) is near the inner wall of the microchannel depicted in Fig. 7(a); the concentration gradually decreases toward the titanium bulk region. The constitution of the equilibrium phase(s) corresponding to the maximum aluminum concentration was double-phase [alpha- $\mathrm{Ti}+\mathrm{TiAl}]$ at the sintering temperature of $1473 \mathrm{~K}$, and was double-phase [ $\mathrm{Ti}_{3} \mathrm{Al}+\mathrm{TiAl}$ ] below $1437 \mathrm{~K}$ (see Fig. 8). Therefore, the structure of the microchannel lining was considered to be comprised of $\mathrm{Ti}_{3} \mathrm{Al}$,
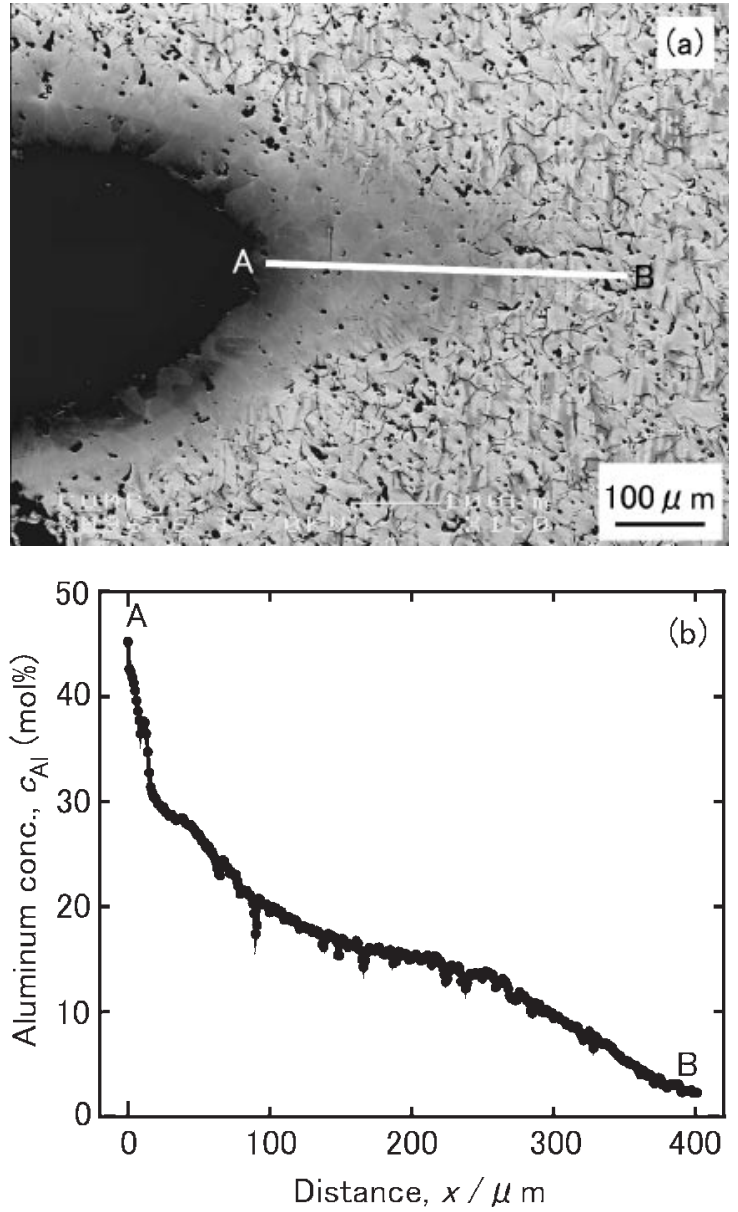

Fig. 7 Back-scattered electron image (a) and aluminum-concentration profile (b) near the microchannel in the Ti-Al specimen.

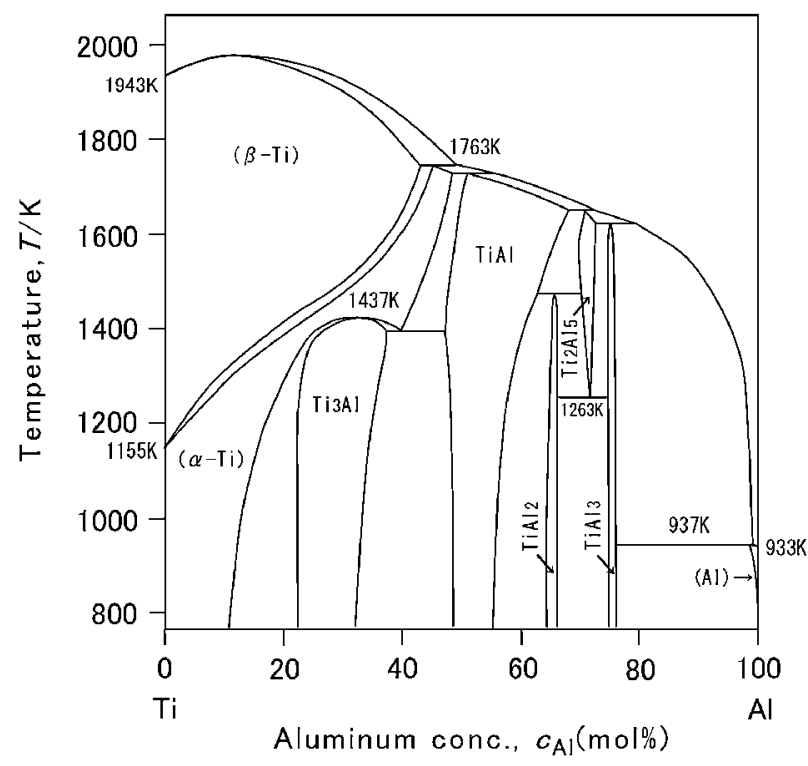

Fig. 8 Ti-Al binary alloy phase diagram. ${ }^{9)}$

TiAl, or supersaturated solid solution.

The maximum aluminum concentration was $17 \mathrm{~mol} \%$ when the binder to metal volume ratio of the sacrificial-core compound was $7: 2 .{ }^{10}$ ) The above results suggest that aluminum concentration of the lining layer increases as the 


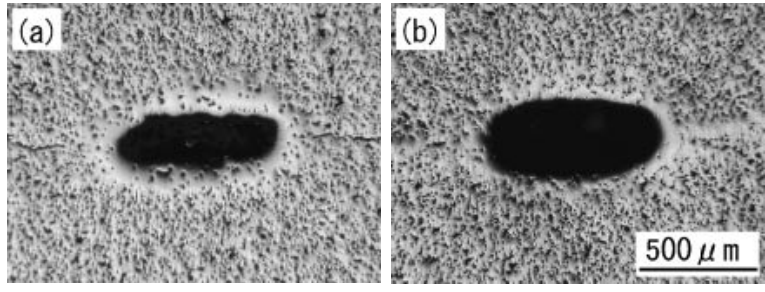

Fig. 9 Optical micrographs showing the effect of third-element addition to the sacrificial core metal on the formation of the microchannel. Combination of body metal and sacrificial-core metal: (a) Ti and Al-17 mol\% Cu, (b) Ti and Al-34 mol\% Cu.
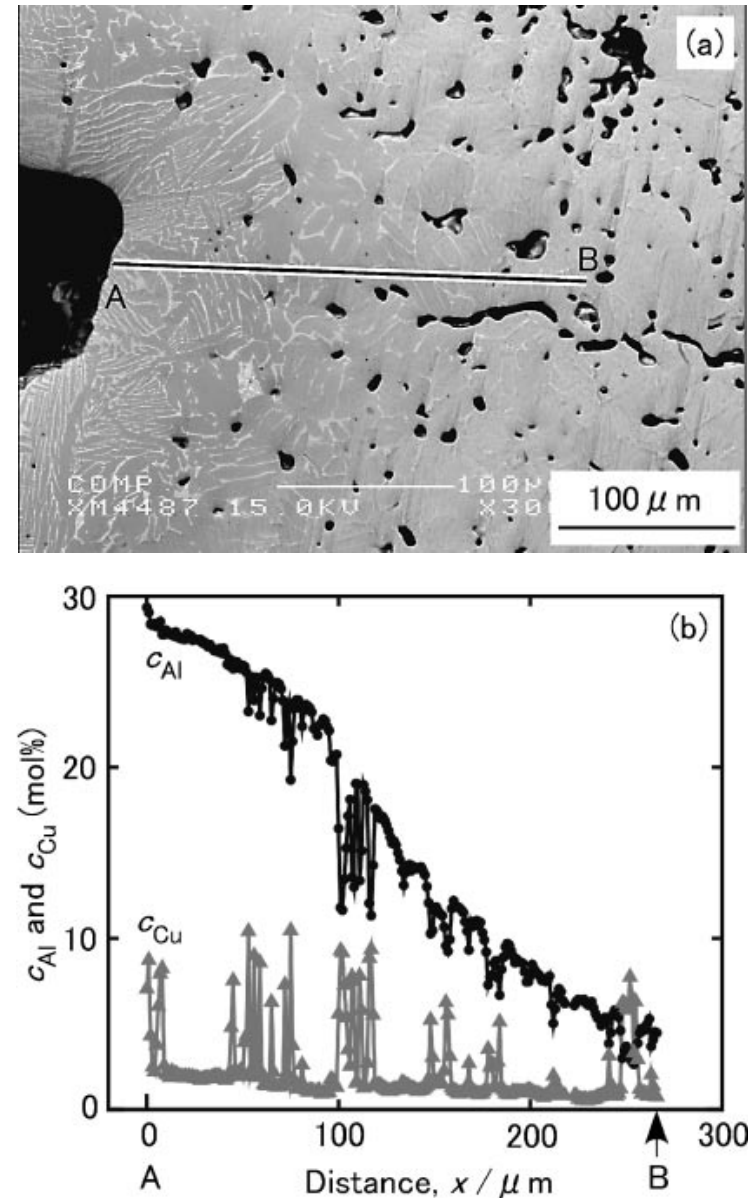

Fig. 10 Back-scattered electron image (a) and aluminum- and copperconcentration profiles (b) near the microchannel in the Ti-Al-Cu specimen produced with Al-17 mol\% Cu sacrificial-core metal.

volume ratio of aluminum powder in the sacrificial core increases.

\subsection{Effects of third-element addition}

Figures 9, 10 and 11 depict the results of the experiments on the effects of third-element addition to the sacrificial core. Figure 9 presents optical micrographs of cross sections of the microchannels fabricated using $\mathrm{Al}-17 \mathrm{~mol} \% \mathrm{Cu}$ and $\mathrm{Al}-$ $34 \mathrm{~mol} \% \mathrm{Cu}$ sacrificial-core metals. Microchannels formed in both specimens, as indicated in these photographs. The cross-sectional contours of the microchannels in Figs. 6(a), 9(a) and 9(b) reveal that the copper addition to the sacrificial-
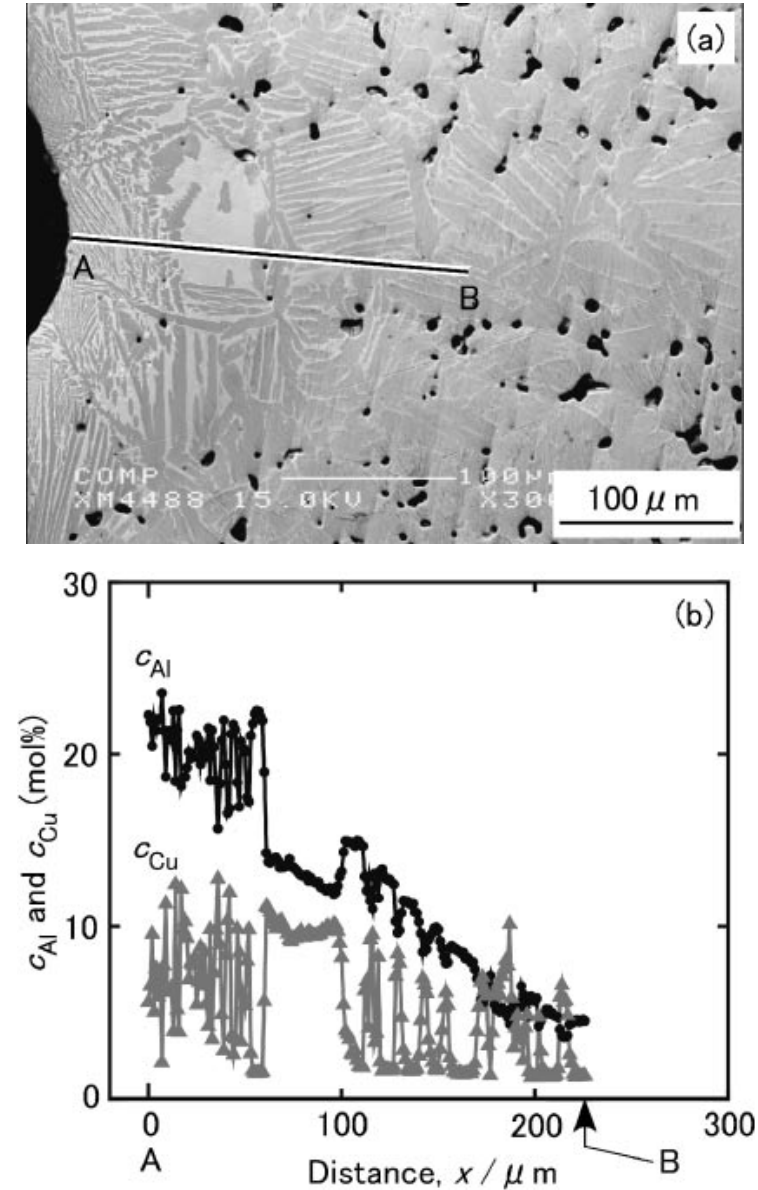

Fig. 11 Back-scattered electron image (a) and aluminum- and copperconcentration profiles (b) near the microchannel in the Ti-Al-Cu specimen produced with Al-34 mol\% Cu sacrificial-core metal.

core metal increases smoothness of the inner wall of the microchannel, which will be a significant factor in practical applications. These results may be caused by dissolution of titanium into copper-rich Al-Cu alloy melt. However, the detailed mechanism is not clear. Further investigation is necessary to explain it.

Figure 10 presents a back-scattered electron image of the lining layer (a) and an EPMA result for the case of Al$17 \mathrm{~mol} \% \mathrm{Cu}$ sacrificial-core metal. Figure 11 shows the case of Al-34 mol\% Cu sacrificial-core metal. A bright copper-rich phase is observed in the microchannel lining in each specimen. This phase has an almost constant copper concentration, $10 \mathrm{~mol} \%$. The aluminum concentration of this phase, however, decreases with the distance from the microchannel, $x$. No such phase has been reported in the Al-Cu-Ti ternary phase diagrams. ${ }^{12,13)}$

The average copper concentrations in the region from $x=$ 0 to $200 \mu \mathrm{m}$ were $2.46 \mathrm{~mol} \%$ and $5.83 \mathrm{~mol} \%$ in Figs. 10(b) and 11(b). These results clearly indicate that the content of the sacrificial-core metal influenced the composition of the microchannel lining.

\section{Conclusions}

We investigated a powder-metallurgical process for fabricating free-form microchannels and lining alloy layers 
in metal bodies, and we examined its feasibility for some metal systems. The results of our investigation can be summarized as follows.

(1) The microchannels formed in sintered powder compacts of titanium, nickel, copper and iron using a sacrificial core compound consisting of a metal powder and an organic binder.

(2) The composition, structure and inner-wall smoothness of the microchannel lining in the sintered titanium specimen can be changed by copper addition to the sacrificial-core metal.

\section{Acknowledgement}

Part of this work was supported by Hosokawa Powder Technology Foundation.

\section{REFERENCES}

1) J. Yoshida: Microreactor Technology, (NTS, Tokyo, 2005) pp. 3-10.

2) R. J. Phillips: Advances in Thermal Modeling of Electronic Compo- nents and Systems, Vol. 2, ed. by A. Bar-Cohen and A. D. Kraus, (ASME, 1990) pp. 109-184.

3) I. Papautsky, A. B. Frazier and H. Swerdlow: Proc. MEMS '97, (IEEE, Nagoya, 1997) pp. 317-322.

4) E. V. Rebrov, M. H. J. M. de Croon and J. C. Schouten: Catalysis Today 69 (2001) 183-192.

5) Micromachine Center $\&$ The Society of $\mu \mathrm{M}$ Frontier: Micromachine Revolution, (The Nikkan Kogyo Shinbun, Tokyo, 1986) p. 67.

6) K. Hosokawa: Microreactors, Epoch-making Technology for Synthesis, ed. by J. Yoshida, (CMC Publication, Tokyo, 2003) pp. 37-46.

7) T. Ohmi, M. Sakurai, K. Matsuura and M. Kudoh: Proceedings of the 15th International Symposium on Transport Phenomena, (CD-ROM) (2004) 364-367.

8) T. Ohmi, K. Matsuura and M. Kudoh: International Journal of SelfPropagating High-Temperature Synthesis, 13-2 (2004) 121-129.

9) S. Nagasaki and M. Hirabayashi: Binary Alloy Phase Diagrams, (AGNE Technical Center, Tokyo, 2001).

10) T. Ohmi, M. Takatoo, M. Iguchi, K. Matsuura and M. Kudoh: Journal of JSEM 5 (2005) 234-238.

11) H. Okamoto: Desk Handbook: Phase Diagrams for Binary Alloys, (ASM International, 2000).

12) Q. Ran and H. H. Stadelmaier: Ternary Alloys, Vol. 5, ed. by G. Petzow and G. Effenberg, (VCH Verlagsgesellschaft, Weinheim, 1992) pp. 5166.

13) P. Villars, A. Prince and H. Okamoto: Handbook of Ternary Alloy Phase Diagrams, Vol. 3, (ASM International, 1995) pp. 3370-3383. 